http://dx.doi.org/10.32929/2446-8355.2020v29n2p157-168

\title{
COMPOSIÇÃO MINERAL DAS FOLHAS DE PESSEGUEIRO SOB A INFLUÊNCIA DA ADUBAÇÃO NITROGENADA DE CRESCIMENTO
}

\author{
Letícia Vanni Ferreira $^{1 *}$, Luciano Picolotto ${ }^{2}$, Juliano Dutra Schmitz ${ }^{3}$, Ivan dos Santos Pereira ${ }^{4}$, \\ Luis Eduardo Corrêa Antunes ${ }^{5}$

\footnotetext{
${ }^{1}$ Doutora em Agronomia/Fruticultura de Clima Temperado, Universidade Federal de Pelotas, Pelotas, RS. * Email do autor correspondente: letivf@hotmail.com

${ }^{2}$ Docente, Coordenadoria Especial de Ciências Biológicas e Agronômicas, Universidade Federal de Santa Catarina, Campus Curitibanos, Curitibanos, SC.

${ }^{3}$ Doutor em Agronomia/Fruticultura de Clima Temperado, Universidade Federal de Pelotas, Pelotas, RS

${ }^{4}$ Pesquisador, Fruticultura de Clima Temperado, Embrapa Clima Temperado, Pelotas, RS.
}

Recebido: 26/02/2018; Aceito: 27/03/2020

\begin{abstract}
RESUMO: Teores de nutrientes foliares indicam o estado nutricional da planta e auxiliam na recomendação de adubação. Além disso, plantas bem nutridas apresentam um adequado desenvolvimento vegetativo, o que resulta em boa produtividade de frutos. Objetivou-se, com o presente trabalho, avaliar a composição foliar de macro e micronutrientes de pessegueiros em crescimento, cultivados em alta densidade de plantio, submetidos a distintas doses de nitrogênio. O trabalho foi conduzido na área experimental da Embrapa Clima Temperado, entre 2012 e 2015. O delineamento experimental foi o de blocos casualizados em parcelas subdivididas segundo fatorial 2 x 4: duas seleções ('Cascata 1067' e 'Cascata 1513') e quatro doses de $\mathrm{N}$ baseadas em valores da comissão de química e fertilidade do solo $(0,30,60$ e 120 $\mathrm{kg} \mathrm{N} \mathrm{ha}{ }^{-1}$ ), conduzidas em quatro repetições, constituídas por quatro plantas. Foi avaliada a concentração foliar de macro e micronutrientes nos anos de 2012, 2013 e 2014. A aplicação de $\mathrm{N}$ em pomares adensados aumenta o teor do nutriente nas folhas. Os níveis de nitrogênio influenciam o estado nutricional das plantas cultivadas em alta densidade. A adubação nitrogenada até $120 \mathrm{~kg} \mathrm{ha}^{-1}$ é insuficiente para manter os teores foliares de $\mathrm{N}$ em níveis considerados normais no terceiro ano após o plantio.
\end{abstract}

Palavras-chave: Prunus persica. Nitrogênio. Nutrição. Densidade de plantio. Nutrientes.

\section{MINERAL COMPOSITION OF THE PEACH LEAVES UNDER THE INFLUENCE OF NITROGEN FERTILIZATION}

\begin{abstract}
Leaf nutrient contents indicate the nutritional status of the plant and aid in the fertilization recommendation. In addition, well-nourished plants have an adequate vegetative development, which results in good fruit yield. The objective of this work was to evaluate macro and micronutrient leaf composition in peach trees cultivated at high planting density, submitted to different nitrogen doses. The essay was conducted in the Embrapa Clima Temperado at experimental area among 2012 and 2015. The combination of the advanced selection of peach trees 'Cascata 1513' and 'Cascata 1067' and doses of nitrogen fertilization $\left(0,30,60\right.$ and $120 \mathrm{~kg}$ of $\left.\mathrm{N} \mathrm{ha}^{-1}\right)$. The foliar concentration of nutrients was evaluated. The
\end{abstract}


application of $\mathrm{N}$ in high density orchards increases the nutrient content in the leaves. Nitrogen fertilization up to $120 \mathrm{~kg} \mathrm{ha}^{-1}$ is insufficient to maintain leaf $\mathrm{N}$ levels at levels considered normal in the third year after planting.

Key words: Prunus persica. Nitrogen. Nutrition. Planting density. Nutrients.

\section{INTRODUÇÃO}

Teores de nutrientes foliares indicam o estado nutricional da planta e auxiliam na recomendação de adubação, em plantas frutíferas (MAYER et al., 2015). O nitrogênio (N) é o nutriente considerado de maior importância para o pessegueiro (FERREIRA et al., 2016) e é aplicado em praticamente todos os pomares desta cultura, nas diferentes regiões produtoras do mundo; é um elemento crítico para a vida da planta, pois compõe aminoácidos, proteínas, enzimas, ácidos nucléicos, clorofila (JOHNSON, 2008) e ácidos orgânicos (FERREIRA et al., 2017).

Há expectativas crescentes da sociedade para que a gestão da nutrição mineral seja realizada de forma ecologicamente correta (CARRANCA et al., 2018). A prática utilizada no mundo todo para determinar o estado nutricional das frutíferas é a análise foliar de nutrientes (JOHNSON et al., 2006). A dose de nitrogênio é recomendada de acordo com o seu teor total na folha, sendo que a aplicação do nutriente deverá ser parcelada durante a plena floração e após o raleio e colheita de frutas, e variam de acordo com o teor de matéria orgânica do solo, com a idade do pomar (CQFS RS/SC, 2016) e com o genótipo, mas também tendo como referência o crescimento e a produtividade (FERREIRA et al., 2018). O crescimento, segundo estes últimos autores, aumenta de forma linear com o acréscimo de nitrogênio. A produtividade também, geralmente, melhora com aplicação de nitrogênio, embora Ferreira $e t$ al. (2018) destacaram que o número de frutos pode ser alterado por diferentes doses do referido nutriente.

Em frutíferas de caroço, os teores de nutrientes foliares em plantas adultas variam em função da cultivar-copa, porta-enxerto, idade da folha, produtividade da planta, condições edafoclimáticas e técnicas culturais aplicadas (COUVILLON, 1982; KNOWLES et al., 1984; BROWN; CUMMINS, 1989; BOYHAN et al., 1995; ZARROUK et al., 2005; LEONEL et al., 2011; ROMBOLÀ et al., 2012; REIGHARD et al., 2013). Neste sentido, a adubação é uma prática que visa corrigir deficiências e proporcionar às plantas condições adequadas de nutrição e, consequentemente, garantir produções economicamente viáveis.

Outro fator correlacionado com a produção em pomares de pessegueiro é a densidade de plantio, conforme também já descrito por Mayer et al. (2016). Neste contexto, a tendência da fruticultura moderna é o adensamento dos pomares, com incremento nas produtividades dos mesmos, que são cada vez mais produtivos. Por outro lado, estudos voltados para a adequação dos níveis de adubação de crescimento, em pomares como os de alta densidade são escassos.

Dado o exposto, o objetivo do presente trabalho foi avaliar a influência das doses crescentes de adubação nitrogenada de crescimento, no estado nutricional de plantas de 
pessegueiro cultivadas em alta densidade de plantio, nas condições edafoclimáticas de Pelotas-RS.

\section{MATERIAL E MÉTODOS}

O experimento foi implantado a campo, na área experimental pertencente à Embrapa Clima Temperado, Pelotas/RS ( $31^{\circ} 40^{\prime} \mathrm{S}$ e $52^{\circ} 26^{\prime} \mathrm{W}$ e altitude de $70 \mathrm{~m}$ ), sendo conduzido de agosto de 2012 a dezembro de 2016. O clima da região segundo a classificação de Köppen é do tipo "Cfa", ou seja, temperado úmido com verões quentes.

O solo do local é moderadamente profundo, com textura média no horizonte A e argilosa no horizonte B, classificados como Argissolo Vermelho Amarelo (EMBRAPA, 2006). De acordo com a análise de pré-plantio, as características do solo foram as seguintes: pH em água de 4,6 $\mathrm{H}+\mathrm{Al}$ de $3,4 \mathrm{cmol}_{\mathrm{c}} \mathrm{dm}^{3}$, teor de matéria orgânica de 1,3\%, de Ca 1,4 $\mathrm{cmolc}_{\mathrm{c}}$ $\mathrm{dm}^{-3}$, de $\mathrm{Mg}$ 1,4 $\mathrm{cmol}_{\mathrm{c}} \mathrm{dm}^{-3}$, de P 17,9 mg dm${ }^{-3}$ e de $\mathrm{K}$ 75,0 $\mathrm{mg} \mathrm{dm}^{-3}$ (CQFS-RS/SC, 2016). A calagem e a adubação de pré-plantio com fósforo (P) foram realizadas conforme a recomendação do manual de adubação e calagem, sendo realizada um mês e três meses antes do plantio, respectivamente. Aplicaram-se $30 \mathrm{~kg}$ de $\mathrm{P}_{2} \mathrm{O}_{5} \mathrm{ha}^{-1}$, sendo utilizado como fonte o superfosfato triplo, e não foi aplicado potássio, já que o mesmo se encontrava na faixa muito alto, não sendo indicada adubação (CQFS-RS/SC, 2004). O manejo do pomar experimental foi realizado conforme as Normas da Produção Integrada de Pêssegos (FACHINELLO et al., 2003).

Os tratamentos consistiram da adubação nitrogenada de crescimento, a qual foi realizada durante os três primeiros anos após a implantação do pomar, com doses abaixo e acima do recomendado pelo teor de matéria orgânica da análise de solo (CQFS-RS/SC, 2004) que determina valores entre 20 a $80 \mathrm{~kg} \mathrm{~N} \mathrm{ha}^{-1}$. As diferentes doses de $\mathrm{N}(0,30,60$ e $120 \mathrm{~kg} \mathrm{~N}$ $\mathrm{ha}^{-1}$ ) foram fracionadas em cinco aplicações (logo após a brotação; 25 dias após a primeira aplicação; 20 dias após a segunda aplicação; 30 dias após a terceira e 20 dias após a quarta aplicação). A fonte fornecedora de $\mathrm{N}$ foi a ureia.

Para a implantação do experimento foram utilizadas mudas das seleções Cascata 1513 e Cascata 1067 enxertadas sobre o portaenxerto 'Capdeboscq', sendo o pomar manejado em sistema de condução em ipsilon $\mathrm{O}$ espaçamento de plantio utilizado foi de $1,5 \times 5,0 \mathrm{~m}$, densidade de 1333 plantas $\mathrm{ha}^{-1}$. As seleções testadas fazem parte do programa de melhoramento genético de frutas de caroço da Embrapa Clima Temperado.

A composição foliar de macro e de micronutrientes foi determinada em amostras de folhas coletadas, anualmente, no mês de novembro, entre a $13^{\mathrm{a}}$ e a $15^{\mathrm{a}}$ semanas após a plena floração. Foram amostradas 100 folhas completas (limbo com pecíolo) em duas plantas centrais de cada parcela. A coleta das folhas foi realizada na porção média dos ramos do ano, localizados no terço mediano da planta, conforme as recomendações de Freire e Magnani (2014). As amostras foram acondicionadas em sacos de papel identificados e imediatamente enviadas ao Laboratório de Nutrição Vegetal da Embrapa Clima Temperado para análise química. Foram determinados os teores foliares dos macronutrientes nitrogênio $(\mathrm{N})$, fósforo $(\mathrm{P})$, potássio $(\mathrm{K})$, cálcio $(\mathrm{Ca})$ e magnésio $(\mathrm{Mg})$, expressos em \%; e dos micronutrientes ferro 
$(\mathrm{Fe})$, manganês $(\mathrm{Mn})$, zinco $(\mathrm{Zn})$, cobre $(\mathrm{Cu})$ e boro $(\mathrm{B})$, expressos em $\mathrm{mg} \mathrm{kg}^{-1}$, de acordo com as metodologias definidas pela Rede Oficial de Laboratórios de Análise de Solo e de Tecido Vegetal do RS e de SC (CQFS-RS/SC, 2016).

O delineamento experimental foi o de blocos casualizados em parcelas subdivididas segundo fatorial 2 x 4: duas seleções ('Cascata 1067' e 'Cascata 1513') e quatro doses de N $\left(0,30,60\right.$ e $\left.120 \mathrm{~kg} \mathrm{~N} \mathrm{ha}^{-1}\right)$, conduzidas em quatro repetições, constituídas por quatro plantas. Os anos de avaliação não foram considerados fatores de tratamento, sendo cada ano analisado separadamente. Os resultados foram submetidos à análise de variância e quando o efeito foi significativo para o fator qualitativo (seleção) realizou-se o teste de Tukey a $5 \%$ de probabilidade de erro. Quando significativo para o fator quantitativo (doses de $\mathrm{N}$ ), foi realizada a análise de regressão a 5\% de probabilidade de erro. As análises estatísticas foram realizadas com o programa SISVAR versão 5.1 (FERREIRA, 2014).

\section{RESULTADOS E DISCUSSÃO}

O fator genótipo não influenciou nos teores de macronutrientes foliares (Tabela 1). No entanto, os teores de $\mathrm{N}$ na folha aumentaram de forma linear com os tratamentos de adubação nos dois primeiros anos de avaliação (Figura 1). Estes resultados corroboram com os obtidos por Brunetto et al. (2007), Pereira et al. (2013), Teixeira (2013) e Sete et al. (2015), em estudos com pessegueiro, em que foram testadas as doses: $40,80 \mathrm{e} 160 \mathrm{~kg} \mathrm{de} \mathrm{N} \mathrm{ha}^{-1}$ ano ${ }^{-1}$; 0 , 22, 44, 66 e $88 \mathrm{~kg} \mathrm{ha}^{-1}$ de $\mathrm{N}$ e 40,80,120, 160, 200 e $240 \mathrm{~kg}$ de $\mathrm{N} \mathrm{ha}^{-1}$, respectivamente. Conforme estabelecido pelo CQFS-RS/SC (2016), o nível de $\mathrm{N}$ foliar considerado normal para o pessegueiro, nos Estados do Rio Grande do Sul e Santa Catarina, é na faixa de 3,30 a $4,50 \%$.

No presente trabalho, observou-se que o teor foliar de $\mathrm{N}$ das plantas do tratamento testemunha não atingiu o nível considerado normal nos três anos de avaliação. Porém, para as demais doses, os níveis de $\mathrm{N}$ foliar encontraram-se dentro da faixa de suficiência considerada normal durante os dois primeiros anos de cultivo. Por outro lado, no terceiro ano de cultivo, os teores foliares foram considerados insuficientes, independente da dose aplicada (Figura 1). Desta forma, é possível inferir que a adubação nitrogenada na fase de crescimento, atualmente recomendada para o pessegueiro no terceiro ano, não atende à demanda requerida pela cultura para pomares adensados.

Atualmente, a maior dose de $\mathrm{N}$ recomendada para plantas de pessegueiro no terceiro ano é de $80 \mathrm{~kg} \mathrm{ha}^{-1}$, quando a matéria orgânica do solo estiver em teores $\leq 2,5 \%$ (CQFS $\mathrm{RS} / \mathrm{SC}, 2016)$. Essa quantidade de $\mathrm{N}$ corresponde apenas a $66,7 \%$ da maior dose utilizada no presente estudo (120 kg ha-1) e que não foi suficiente para elevar os teores foliares de $\mathrm{N}$ à faixa considerada normal. No entanto, a mineralização da matéria orgânica e, consequentemente, a resposta do pessegueiro à adubação nitrogenada, segundo Brunetto et al. (2007) depende, dentre outros, de fatores edafoclimáticos, com destaque para a umidade no solo e a temperatura. 
Tabela 1. Teores de macronutrientes (N, P, K, Ca e $\mathrm{Mg}$ ) e micronutrientes ( $\mathrm{Fe}, \mathrm{Mn}, \mathrm{Zn}$ e $\mathrm{Cu}$ ) em folhas de genótipos de pessegueiros (Prunus persica L.) em função de diferentes doses de N em adubação de crescimento, nos anos de 2012, 2013 e 2014. Embrapa Clima Temperado, Pelotas, 2020. Content of macronutrients ( N, P, K, Ca and Mg) and micronutrients (Fe, Mn, $\mathrm{Zn}$ and $\mathrm{Cu}$ ) in leaves of peach (Prunus persica L.) genotypes as a function of different doses of $N$ in growth fertilizer, in years 2012, 2013 and 2014. Embrapa Clima Temperado, Pelotas, 2020 .

\begin{tabular}{|c|c|c|c|c|c|}
\hline \multicolumn{6}{|c|}{2012} \\
\hline & $\mathbf{N}(\%)$ & $\mathbf{P}(\%)$ & $\mathbf{K}(\%)$ & $\mathrm{Ca}(\%)$ & $\operatorname{Mg}(\%)$ \\
\hline \multicolumn{6}{|l|}{ Genótipo } \\
\hline Cascata 1067 & $3,45^{\mathrm{ns}}$ & $0,33^{\mathrm{ns}}$ & $1,89^{\mathrm{ns}}$ & $1,04^{\mathrm{ns}}$ & $0,53^{\mathrm{ns}}$ \\
\hline Cascata 1513 & 3,50 & 0,33 & 1,91 & 1,13 & 0,54 \\
\hline $\operatorname{CV}(\%)^{1}$ & 7,13 & 7,63 & 7,26 & 16,22 & 14,62 \\
\hline \multicolumn{6}{|c|}{2013} \\
\hline \multicolumn{6}{|l|}{ Genótipo } \\
\hline Cascata 1067 & $3,19 \mathrm{~b}$ & $0,42^{\mathrm{ns}}$ & $2,44^{\mathrm{ns}}$ & $1,50^{\mathrm{ns}}$ & $0,40^{\mathrm{ns}}$ \\
\hline Cascata 1513 & $3,41 \mathrm{a}$ & 0,39 & 2,41 & 1,48 & 0,40 \\
\hline $\mathrm{CV}(\%)^{1}$ & 6,96 & 13,52 & 10,98 & 12,77 & 14,39 \\
\hline \multicolumn{6}{|c|}{2014} \\
\hline \multicolumn{6}{|l|}{ Genótipo } \\
\hline Cascata 1067 & $2,69^{\mathrm{ns}}$ & $0,33^{\mathrm{ns}}$ & $2,01^{\mathrm{ns}}$ & $1,67^{\mathrm{ns}}$ & $0,55^{\mathrm{ns}}$ \\
\hline Cascata 1513 & 2,90 & 0,32 & 2,01 & 1,62 & 0,56 \\
\hline \multirow[t]{2}{*}{ CV(\%) } & 10,89 & 18,60 & 8,21 & 15,30 & 12,85 \\
\hline & & 2012 & & & \\
\hline Genótipos & $\mathrm{Fe}$ & Mn & $\mathbf{Z n}$ & $\mathbf{C u}$ & \\
\hline \multicolumn{6}{|c|}{...................mg kg ${ }^{-1} \ldots \ldots$} \\
\hline Cascata 1067 & $69,12^{\mathrm{ns}}$ & $66,56^{\mathrm{ns}}$ & $13,25^{\mathrm{ns}}$ & $6,00^{\mathrm{ns}}$ & \\
\hline Cascata 1513 & 65,31 & 66,75 & 13,56 & 6,25 & \\
\hline C.V.(\%) & 9,97 & 29,58 & 13,85 & 10,54 & \\
\hline \multicolumn{6}{|c|}{2013} \\
\hline \multicolumn{6}{|l|}{ Genótipos } \\
\hline Cascata 1067 & $68,12^{\mathrm{ns}}$ & & & $14,62 \mathrm{~b}$ & $9,56^{\mathrm{ns}}$ \\
\hline Cascata 1513 & 68,25 & & & $17,12 \mathrm{a}$ & 9,68 \\
\hline C.V. $(\%)^{1}$ & 13,69 & & & 10,45 & 5,94 \\
\hline \multicolumn{6}{|c|}{2014} \\
\hline \multicolumn{6}{|l|}{ Genótipos } \\
\hline Cascata 1067 & $77,19^{\mathrm{ns}}$ & & & $19,00^{\mathrm{ns}}$ & $8,44^{\mathrm{ns}}$ \\
\hline Cascata 1513 & 71,44 & & & 16,15 & 8,68 \\
\hline C.V. $(\%)^{1}$ & 16,54 & & & 35,94 & 14,30 \\
\hline
\end{tabular}

${ }^{1}$ C.V.(\%) Coeficiente de variação.

*Médias seguidas por letras distintas, minúsculas na coluna, diferem entre si pelo Teste de Tukey a $5 \%$ de probabilidade de erro. *ns (não significativo) a 5\% de probabilidade de erro.

Fonte: Autoria própria. Own authorship. 

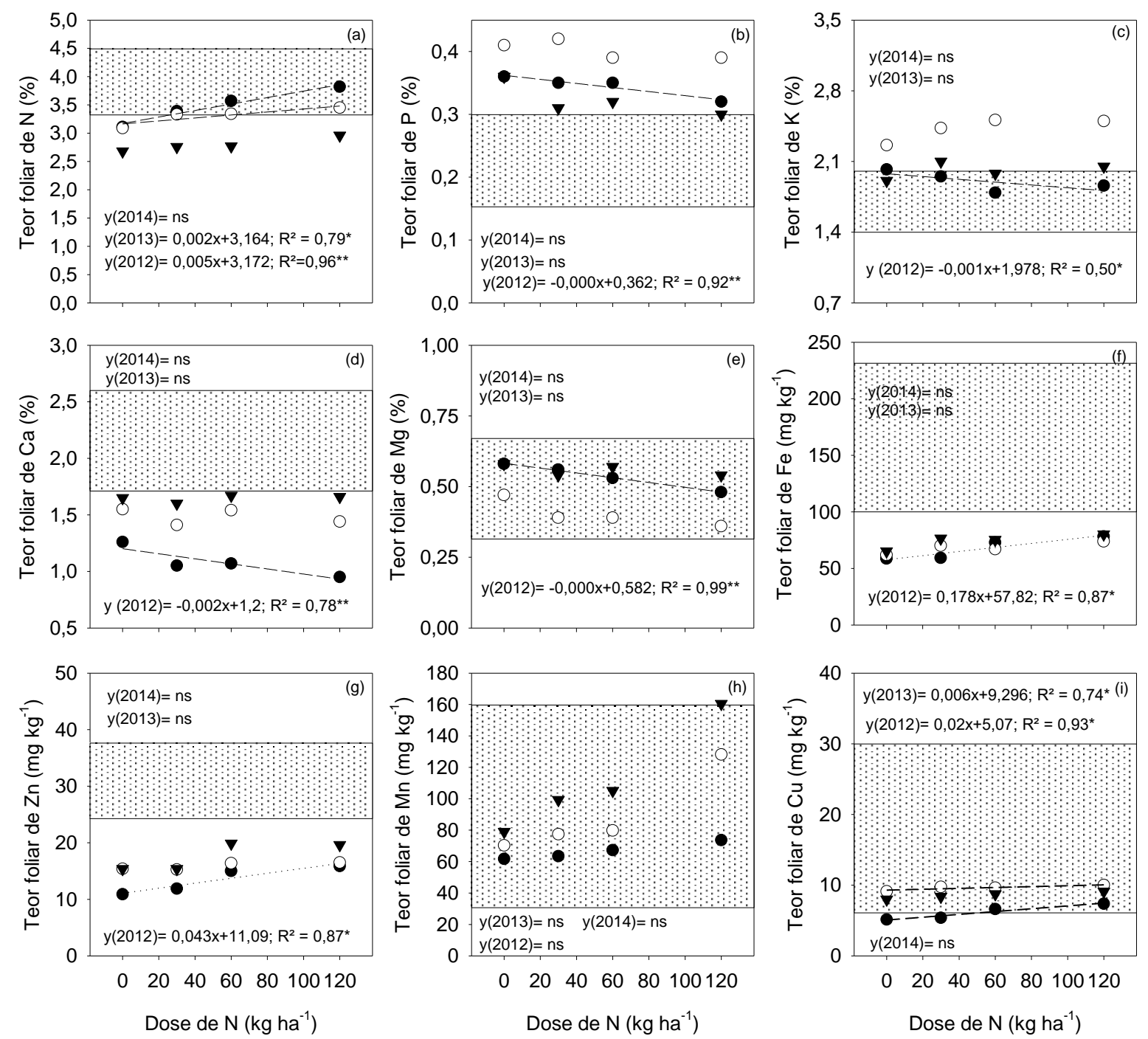

- $2012 \quad 0 \quad 2013 \quad \nabla 2014$

Faixa correspondente ao teor foliar Normal (CQFS-RS/SC, 2016)

**, * significativo pelo teste $\mathrm{F}$ a $1 \%$ e $5 \%$ de probabilidade de erro, respectivamente. Linhas pontilhadas representam os limites dos teores dos nutrientes dentro da normalidade (LS limite superior, LI limite inferior) de acordo com CQFS-RS/SC (2016). **, * significant by the F test at 1\% and 5\% probability of error, respectively. Dotted lines represent the limits of the nutrient content within the normal range (LS upper limit, LI lower limit) according to CQFS-RS/SC (2016).

Figura 1. Teores foliares de nitrogênio-N (a), fósforo-P (b), potássio-K (c), cálcio-Ca (d), magnésio-Mg (e), ferro (f), zinco-(g), manganês-Mn (h) e cobre-Cu (i), em 2012, 2013 e 2014 em pessegueiros (Prunus persica L.) das Seleções 'Cascata 1067' e 'Cascata 1513' submetidas a diferentes doses de adubação nitrogenada de crescimento. Leaf contents of nitrogen- $N(a)$, phosphorus- $P(b)$, potassium- $K(c)$, calcium-Ca (d), magnesium-Mg (e), iron $(f)$, zinc- $(g)$, manganese -Mn (h) and copper-Cu (i), in 2012, 2013 and 2014 in peach trees (Prunus persica L.) from the Selections 'Cascata 1067' and 'Cascata 1513' submitted to different doses of nitrogen growth fertilizer.

Fonte: Autoria própria. Own authorship. 
Acredita-se que o baixo teor de matéria orgânica no solo da área experimental $(1,3 \%)$ possa ter contribuído para uma menor quantidade de $\mathrm{N}$ na folha. Chiodini et al. (2013) destacam que a manutenção da matéria orgânica pode afetar direta ou indiretamente a disponibilidade e a absorção de nutrientes pelas culturas. As perdas por lixiviação e volatilização também, segundo Sete et al. (2015), podem afetar a quantidade ofertada de N às plantas.

Para Chiodini et al. (2013), os níveis adequados de matéria orgânica são benéficos ao solo de várias formas e a maioria dos benefícios, ocorrem em função dos produtos liberados à medida que os resíduos orgânicos são decompostos no solo. De acordo com os mesmos, a matéria orgânica contém elementos essenciais para as plantas como boro, enxofre, fósforo e nitrogênio. Desta forma, pode-se sugerir que em pomares de alta densidade e na condição experimental atual, a adubação nitrogenada deve ser suplementada. Porém, estudos específicos devem ser conduzidos a fim de que sejam definidas as quantidades de $\mathrm{N}$ necessária às plantas levando em consideração níveis de matéria orgânica mais ampla, além dos já verificados pela pesquisa.

Provavelmente, a maior demanda de $\mathrm{N}$ em pomares de alta densidade, ocorra pelo maior acúmulo de massa seca (raízes, folhas, troncos) por hectare, em comparação a pomares de menor densidade, já que segundo Mayer et al. (2016), avaliando as densidades de 1.587 1.058, 794 pl.ha ${ }^{-1}, 635,529$ pl.ha $^{-1}$ verificaram crescimento semelhante entre as plantas, o que indica maior quantidade de massa vegetal desenvolvida por área.

Os seguintes níveis de macronutrientes foliares são considerados normais: $\mathrm{P}(0,15-0,28$ $\%), \mathrm{K}(1,31-2,06 \%)$, Ca (1,64-2,61 \%) e Mg (0,52-0,83 \%) (CQFS RS/SC, 2016). Os teores de P, K, Ca e Mg foliares em 2012, não tiveram influência do genótipo. Entretanto, foram inversamente proporcionais ao $\mathrm{N}$ aplicado no solo (Figura 1), concordando com os resultados obtidos por Amorim (2012), que observou relação inversa entre a dose de $\mathrm{N}$ aplicada e os teores foliares de macronutrientes. Segundo Pereira et al. (2015), como o N é o nutriente presente em maior concentração, ocorre uma estreita relação entre a adubação com $\mathrm{N}$ e a absorção de outros nutrientes. A diminuição dos teores foliares de alguns nutrientes pode ter relação com alterações químicas ocorridas no solo ao longo do tempo.

Leal et al. (2007), comparando doses de ureia na implantação e formação de pomares de caramboleira, não verificaram no primeiro ano de cultivo efeito da adubação nitrogenada sobre os atributos químicos do solo. Já no segundo e terceiro ano, estes atributos químicos do solo tiveram modificações. Com aumento das doses de $\mathrm{N}$ verificaram redução do $\mathrm{pH}$ solo, o qual atribuíram a reações que produzem $\mathrm{H}^{+}$(nitrificação). Destacam ainda que quanto mais baixo o valor de $\mathrm{pH}$, menor a concentração de $\mathrm{K}, \mathrm{Ca}$ e $\mathrm{Mg}$ no solo e, consequentemente, menor a soma de bases e o índice de saturação por bases. Possivelmente, essa diluição na disponibilidade nutricional tenha ocorrido no presente trabalho afetando a absorção pela planta e, consequentemente, acúmulo nas folhas conforme se aumentou a dose de $\mathrm{N}$. Elementos como, por exemplo, o Mg constituem parte da molécula de clorofila, atuando como ativador de diversas enzimas e correlaciona-se positivamente com o crescimento dos ramos (BOYHAN et al., 1995; JOHNSON, 2008). Assim, sua deficiência poderia afetar o crescimento adequado da planta. 
Os níveis de cálcio foram menores nas plantas que receberam $\mathrm{N}$, quando comparados aos das plantas do tratamento testemunha, o que está de acordo com o verificado Leitske et al. (2015) quando avaliaram diferentes doses de $\mathrm{N}$ para a cultura do mirtileiro. Carvalho et al. (2002) estudando teores foliares de nutrientes, mencionam que o decréscimo no teor de $\mathrm{Ca}$ provavelmente ocorre devido a uma provável competição entre os íons provenientes da hidrólise da ureia aplicado ao solo e o $\mathrm{Ca}^{++}$. Por outro lado, cabe salientar que deficiências de Ca geralmente não são comuns em pessegueiro (JOHNSON, 2008). Já o P, não é limitante ao desenvolvimento das plantas e à produção da cultura. De acordo com o CQFS RS/SC (2016), não é observada resposta do pessegueiro à aplicação de fertilizante fosfatado na região Sul do Brasil, quando o teor de $\mathrm{P}$ foliar é maior do que $0,09 \%$.

Observou-se relação inversa dos níveis de $\mathrm{K}$ nas folhas e a dose de $\mathrm{N}$ aplicada no solo (Figura 1), corroborando com o verificado por Leal et al. (2007). No entanto, estes resultados não concordam com os verificados por Sete et al. (2015). Segundo Dolinski et al. (2005), a resposta à adubação nitrogenada variou entre os anos, sendo influenciada, dentre outros fatores, pelas condições climáticas e de manejo do pomar. O K é um elemento que normalmente se encontra com teores normais ou acima do normal, devido a riqueza deste nutriente do material de origem dos solos gaúchos onde o pessegueiro é cultivado (FREIRE e MAGNANI, 2002), além de ser um nutriente de baixa demanda na fase de formação das plantas (ROMBOLÀ et al. 2012).

Em 2014 não foram observadas diferenças estatísticas. Em todos os tratamentos, verificou-se que o $\mathrm{N}$ foliar estava abaixo da normalidade, o que pode ser explicado pela elevada precipitação média $(1862,9 \mathrm{~mm})$ neste ano, que pode ter ocasionado perdas de nutrientes por lixiviação. De acordo com Dolinski et al. (2005), os teores foliares de nutrientes podem variar entre os anos, evidenciando possíveis relações entre os teores foliares desses elementos e as condições climáticas.

Para micronutrientes, os seguintes valores são considerados normais para análises foliares em pessegueiro e nectarineira (CQFS RS/SC, 2016): Cu (6-30 mg.kg-1), Fe (100-230 mg. $\left.\mathrm{kg}^{-1}\right)$, Mn (31-160 mg. $\left.\mathrm{kg}^{-1}\right)$ e Zn (24-37 mg.kg- ${ }^{-1}$.

Verificou-se influência da adubação nitrogenada nos micronutrientes (Figura 1), embora a grande maioria tenha ficado em valores considerados normais, concordando com Dolinski et al. (2005) os quais não observaram diferenças significativas entre as doses de $\mathrm{N}$ testadas. Todavia, segundo estes autores, os teores de micronutrientes como, por exemplo, Zn e B variaram entre os anos, evidenciando possíveis relações entre os teores foliares desses elementos e as condições meteorológicas. Dentre os micronutrientes, o principal efeito do $\mathrm{Fe}$ na planta é a transferência de energia durante o processo de fotossíntese e respiração; O Zn atua na formação das auxinas, e sua falta causa a desordem conhecida como "folha pequena", encontrada em quase todas as regiões produtoras mundiais. Já o B é essencial para o crescimento e desenvolvimento da planta; atua no crescimento do tubo polínico e do meristema, transporte de açúcares, síntese da parede celular, produção de fitohormônios e integridade da membrana celular (MAYER et al., 2015). 


\section{CONCLUSÃO}

Nas condições em que foi realizado o estudo:

Os níveis de nitrogênio influenciam o estado nutricional das plantas cultivadas em alta densidade;

A adubação nitrogenada até $120 \mathrm{~kg} \mathrm{ha}^{-1}$ é insuficiente para manter os teores foliares de $\mathrm{N}$ em níveis considerados normais no terceiro ano após o plantio.

\section{AGRADECIMENTOS}

À Coordenação de Aperfeiçoamento de Pessoal de Nível Superior (CAPES), CNPq e à Empresa Brasileira de Pesquisa Agropecuária (EMBRAPA) pelo apoio financeiro.

\section{REFERÊNCIAS BIBLIOGRÁFICAS}

AMORIM, D. A. Adubação Nitrogenada e Potássica em Goiabeiras "Paluma' manejadas no sistema intensivo de Produção. 2012. 116 f. Tese (Doutorado em Agronomia-Produção Vegetal) Faculdade de Ciências Agrárias e Veterinárias Unesp, Jaboticabal, 2012.

BOYHAN, G. E.; NORTON, J. D.; PITTS, J. A. Establishment, growth, and foliar nutrient content of plum trees on various rootstocks. HortScience, Alexandria, v. 30, n. 2, p.219-221, 1995.

BROWN, S. K.; CUMMINS, J. N. Rootstock effect on foliar nutrient concentrations of 'Redhaven' peach trees. HortScience, Alexandria, v. 24, n. 5, p.769-771, 1989.

BRUNETTO, G. de MELO, G.W.; KAMINSKI, J.; CERETTA, C.A. Adubação nitrogenada em ciclos consecutivos e seu impacto na produção e na qualidade do pêssego. Pesquisa Agropecuária Brasileira, Brasília, v. 42, n. 12, p.1721-1725, 2007. Disponível em https://seer.sct.embrapa.br/index.php/pab/article/view/7754. Acesso em: 20 fev 2018.

CARRANCA, C.; BRUNETTO, G.; TAGLIAVINI, M. Nitrogen Nutrition of Fruit Trees to Reconcile Productivity and Environmental Concerns. Plants, v. 7, n. 1, p.4, 2018. Disponível em: http://www.mdpi.com/2223-7747/7/1/4. Acesso em 21 fev 2018.

CARVALHO, A. J. C. MONNERAT, P.H.; MARTINS, D.P.; BERNARDO, S.; SILVA, J. A. Teores foliares de nutrientes no maracujazeiro amarelo em função de adubação nitrogenada, irrigação e épocas de amostragem. Scientia Agricola, São Paulo, v. 59, n. 1, p.121-127, 2002. CHIODINI, B. M.; SILVA, A. G.; NEGREIROS, A. B.; MAGALHÃES, L. B. Matéria orgânica e sua influência na nutrição de plantas. Cultivando o Saber, Rio Verde, v. 6, n. 1, p.181-190, 2013.

COMISSÃO DE QUÍMICA E FERTILIDADE DO SOLO - RS/SC. Manual de adubação e calagem para os Estados do Rio Grande do Sul e de Santa Catarina. 10 ed. Porto Alegre: SBCS Núcleo Regional Sul/UFRGS, 2004. 400 p. 
COMISSÃO DE QUÍMICA E FERTILIDADE DO SOLO - RS/SC. Manual de adubação e calagem para os Estados do Rio Grande do Sul e de Santa Catarina. 11. ed. Santa Maria: SBCS. Núcleo Regional Sul, 2016. 375 p.

COUVILLON, G. A. Leaf elemental content comparisons of own-rooted peach cultivars to the same cultivars on several peach seedling rootstocks. Journal of the American Society for Horticultural Science, Mount Vernon, v. 107, n. 4, p.555-558, 1982.

DOLINSKI, M. A. SERRAT, B. M.; MOTTA, A. C. V.; CUQUEL, F. L.; SOUZA, S. R.; MAY-DEMIO, L. L.; MONTEIRO, L. B. Produção, teor foliar e qualidade de frutos do pessegueiro "Chimarrita" em função da adubação nitrogenada, na região da Lapa - PR. Revista Brasileira de Fruticultura, Jaboticabal, v. 27, n .1, p.295-299, 2005.

EMPRESA BRASILEIRA DE PESQUISA AGROPECUÁRIA - EMBRAPA. Sistema brasileiro de classificação de solos. Rio de janeiro: EMBRAPA / CNPS, 2006. 412 p.

FACHINELlO, J. C.; TIBOLA, C. S.; VICENZI, M.; PARISOTTO, E.; PICOLOTTO, L.; MATTOS, M. L. T. Produção integrada de pêssegos: três anos de experiência na região de Pelotas-RS. Revista Brasileira de Fruticultura, Jaboticabal v. 25, n. 2, p.256-258, 2003.

FERREIRA, D.F. Sisvar: a guide for its bootstrap procedures in multiple comparisons. Ciência e Agrotecnolgia, v.38, p.109-112, 2014.

FERREIRA, L. V.; ANTUNES, L. E. C. Crescimento e acúmulo de ácidos orgânicos na seiva mineral, raízes e exsudatos radiculares de portaenxertos de Prunus em função da fertilização nitrogenada. Ciência Agrícola, v. 15, n. 2, p.11-18, 2017.

FERREIRA, L. V.; CORRÊA, A. P. A.; PICOLOTTO, L.; FLORES CANTILlANO, R. F.; ANTUNES, L. E. C. Qualidade de pêssegos submetidos à adubação nitrogenada. Revista Iberoamericana Tecnologia Postcosecha, México, v. 17, n. 2, p.231-240, 2016.

FERREIRA, L. V.; PICOLOTTO, L.; PEREIRA, I. D. S.; SCHIMITZ, J. D.; ANTUNES, L. E. C. Nitrogen fertilization in consecutive cycles and its impact on high-density peach crops. Pesquisa Agropecuária Brasileira, Brasília, v. 53, n. 2, p.172-181, 2018.

FREIRE, C. J. S. Recomendação de adubação potássica de manutenção para a cultura da ameixeira por meio da análise foliar. Pelotas: Embrapa Clima Temperado, 2002. 2p. (Comunicado Técnico, 67).

FREIRE, C. J.; MAGNANI, M. Adubação e correção do solo. In: RASEIRA, M. C. B.; PEREIRA, J. F. M.; CARVALHO, F. L. C. Pessegueiro. Brasília: Embrapa, 2014. cap. 11, p.73-141.

JOHNSON, R.S. Nutrient and water requirements of peach trees. In: LAYNE, D.; BASSI, D. The peach: botany, production and uses. Wallingford: CABI. 2008. p.303-331.

JOHNSON, R. S.; ANDRIS, H.; DAY, K.; BEEDE, R. Using dormant shoots to determine the nutritional status of peach trees. Acta Horticulturae, The Hague, n. 721, p.285-290, 2006.

KNOWLES, J. W.; DOZIER JÚNIOR., W. A.; EVANS, C. E.; CARLTON, C. C.; MCGUIRE, J.M. Peach rootstock influence on foliar and dormant stem nutrient content. 
Journal of the American Society for Horticultural Science, Mount Vernon, v. 109, n. 3, p.440-444, 1984.

LEAL, R. M.; NATALE, W.; PRADO, R. M.; ZACCARO, R. P. Adubação nitrogenada na implantação e na formação de pomares de caramboleira. Pesquisa Agropecuária Brasileira, Brasília, v. 42, n. 8, p.1111-1119, 2007. Disponível em http://www.alice.cnptia.embrapa.br/handle/doc/122785. Acesso em: 22 mai. 2017.

LEITZKE, L. N., PICOLOTTO, L.; DOS SANTOS PEREIRA, I.; VIGNOLO, G. K.; SCHMITZ, J. D.; VIZZOTTO, M.; ANTUNES, L. E. C. Nitrogen fertilizer affects the chemical composition of the substrate, the foliar nutrient content, the vegetative growth, the production and fruit quality of blueberry. Científica, Jaboticabal, v. 43, n. 4, p.316-324, 2015.

LEONEL, S.; SOUZA, M. E.; TECCHIO, M. A.; SEGANTINI, D. M. Leaf nutritional levels in peach and nectarine grown in subtropical climate. Revista Brasileira de Fruticultura, Jaboticabal, v. 33, n. especial, p.752-761, 2011.

MAYER, N. A.; NEVES, T. R.; ROCHA, C. T.; SILVA, V. A. L. Adensamento de plantio em pessegueiros 'Chimarrita'. Revista de Ciências Agroveterinárias, Lages, v. 15, n. 1, p.50-59, 2016. Disponível em: http://dx.doi.org/10.5965/223811711512016050. Acesso em: 12 abr. 2017.

MAYER, N. A.; UENO, B.; SILVA, V. A. L.; Teores de nutrientes foliares de pessegueiro em cinco porta-enxertos. Revista Brasileira de Fruticultura, Jaboticabal, v. 37, n. 4, p.1045$1052,2015$.

PEREIRA, I. D. S.; NAVA, G.; PICOLOTTO, L.; VIGNOLO, G. K.; GONÇALVES, M. A; ANTUNES, L. E. C. Exigência nutricional e adubação da amoreira-preta. Revista de Ciências Agrárias - Amazonian Journal of Agricultural and Environmental Sciences, Amazônia, v. 58, n. 1, p.96-104, 2015. Disponível em https://periodicos.ufra.edu.br/index.php?journal=ajaes\&page=article\&op=view \&path $\% 5 \mathrm{~B} \% 5$ $\mathrm{D}=1755$. Acesso em: 20 mai. 2017.

PEREIRA, I. S.; PICOLOTTO, L.; MESSIAS, R. S.; POTES, M. L.; ANTUNES, L. E. C. Adubação nitrogenada e características agronômicas em amoreira-preta. Pesquisa Agropecuária Brasileira, Brasília, v. 48, n. 4, p.373-380, 2013.

REIGHARD, G. L.; BRIDGES, W.; RAUH, B.; MAYER, N. A. Prunus rootstocks influence peach leaf and fruit nutrient content. Acta Horticulturae, The Hague, n. 984, p.117-124, 2013.

ROMBOlÀ, A. D.; SORRENTI, G.; MARODIN, G. A. B.; PIERI, A, Z.; BARCA, E. Nutrição e manejo do solo em fruteiras de caroço em regiões de clima temperado. Semina: Ciências Agrárias, Londrina, v. 33, n. 2, p.639-654, 2012. Disponível em: http://www.redalyc.org/articulo.oa?id=445744112038. Acesso em: 19 abr. 2017.

SETE, P. B.; MElO, G. W. B.; OLIVEIRA, B. S.; FREITAS, R. F; MAGRO, R. AMBROSINI, V. G.; TRAPP, T.; COMIN, J. J.; GATIBONI, L. C.; BRUNETTO, G. Perdas de nitrogênio do solo e resposta do pessegueiro à adição de composto orgânico. Ciência Rural, Santa Maria, v. 45, n. 4, p.651-657, 2015. 
TEIXEIRA, W. W. R. Nutrição Nitrogenada e Intensidade de Raleio na Produção de Pêssego. 2013. 42 p. Dissertação (Mestrado em Ciência do solo) - Universidade Federal do Paraná, Curitiba, 2013.

ZARROUK, O.; GOGORCENA, Y.; GÓMEZ-APARISI, J.; BETRÁN, J. A.; MORENO, M. A. Influence of almond $x$ peach hybrids rootstocks on flower and leaf mineral concentration, yield and vigour of two peach cultivars. Scientia Horticulturae, Amsterdam, n. 106, p.502514, 2005. 